\title{
Some Extensions of Banach's Contraction Theorem
}

\author{
Philip R. Meyers
}

\author{
(February 2, 1965)
}

\begin{abstract}
The class of self-mappings of a metrizable space which are contractions under at least one complete metric is shown to include a subset of the local contractions. The required metric is constructed in a sequence of steps.
\end{abstract}

\section{Introduction}

The contraction theorem of Banach remains the most fruitful means for proving and analyzing the convergence of iterative processes. For this reason, extensions of the theorem are of continuing interest. The present paper describes some extensions to a class of functions called local contractions.

For completeness, we include here a resumé of the relevant definitions. A metric space $(X, p)$ consists of a nonempty set $X$ and a nonnegative-valued function $p$ defined on $X \times X$ and satisfying

$$
\begin{aligned}
& p(x, y)>0 \text { if } x \neq y, \\
& p(x, x)=0, \\
& p(x, z) \leqslant p(x, y)+p(y, z), \\
& p(x, y)=p(y, x) .
\end{aligned}
$$

Convergence of a sequence $\left\{x_{n}\right\}$ of points in $X$ to a point $x \in X$ is defined by $p\left(x_{n}, x\right) \rightarrow 0$, and is denoted $x_{n} \rightarrow x$. A sequence $\left\{x_{n}\right\}$ is called a Cauchy sequence if, for every fixed $m, p\left(x_{n}, x_{n+m}\right) \rightarrow 0$. If every Cauchy sequence is convergent, then $(X, p)$ is called a complete metric space.

Consider a function $f: X \rightarrow X$. For any $x \in X$, there is defined the sequence of iterates $x, f(x)=x_{1}, f^{2}(x)$ $=f\left(x_{1}\right)=x_{2}$, etc. These can be interpreted as the outputs of successive steps in an iterative process. The sequence of iterates consists of only a single point, if and only if the starting point $x$ is a fixed point of $f$, i.e., $f(x)=x$.

A contraction on $(X, p)$ is a map $f: X \rightarrow X$ for which there exists a constant $\lambda, 0 \leqq \lambda<1$, such that

$$
p(f(x), f(y)) \leqslant \lambda p(x, y)
$$

for every $x, y \in X$. Banach's contraction theorem, of which a proof may be found in $[1],{ }^{1}$ asserts that if $f$ is a contraction on a complete metric space $(X, p)$, then (i) $f$ has a unique fixed point $\xi$, (ii) for every $x \in X$ the sequence of iterates $f^{n}(x) \rightarrow \xi$, and (iii) the convergence is geometric, in fact

$$
p\left(f^{n}(x), \xi\right) \leq \lambda^{n} p(x, \xi) .
$$

The assurance of a geometric rate of convergence is what makes this theorem so valuable in discussing iterative processes. That a function has a fixed point, or even that the iterates of every point converge to the fixed point, is not sufficient information for some applications. If, however, one can show that the function is a contraction, then the theorem can be applied and geometric convergence can be inferred.

In this paper, we show that some functions, which are not contractions on $(X, p)$, can become contractions with suitable new metrics $\bar{p}$ which are topologically equivalent to $p$, i.e., ones for which

$$
\bar{p}\left(x_{n}, x\right) \rightarrow 0 \text { if and only if } p\left(x_{n}, x\right) \rightarrow 0 .
$$

Our general aim is to determine classes of functions $f$ for which such remetrizations are possible. ${ }^{2}$ Note that we want $(X, \bar{p})$ to be complete whenever $(X, p)$ is, so that the contraction theorem will be applicable after the remetrization. Conclusion (i) of that theorem has the same significance for $(X, \bar{p})$ as for $(X, p)$, and the same is true of conclusion (ii) by virtue of $\left(5^{\prime}\right)$, but geometric convergence in $(X, \bar{p})$ need not imply geometric convergence in $(X, p)$ so that some care in interpretation might be required. However, our constructions do in fact preserve "ultimately geometric convergence" of sequences.

\section{Results}

The following definitions are required for the statements and proofs of our results. A function $f: X \rightarrow X$ is a local contraction (l.c.) if there exist real-valued functions $\mu(x), \lambda(x)$, with $\mu(x)>0$ and $0<\lambda(x)<1$, such that whenever $y, z$ are in the sphere

$$
S(x, \mu(x))=\{u: p(x, u) \leqslant \mu(x)\}
$$

${ }^{2}$ I wish to thank A. J. Goldman (NBS Operations Research Section) for first suggesting this problem and for his continuing interest and suggestions as to content and exposition. 
it follows that

$$
p(f(y), f(z)) \leqslant \lambda(x) p(y, z)
$$

If $\mu(x)$ (resp. $\lambda(x))$ is constant, we have a $\mu$-(resp. $\lambda$-) uniform local contraction (u.l.c.). If both are constant we have a $\mu, \lambda$-u.l.c.

A chain $\sigma_{x y}$ is a finite set of points $\left[x=x_{0}, x_{1}, \ldots\right.$, $\left.x_{n}=y\right]$; its length is

$$
L\left(\sigma_{x y}\right)=\sum_{i=1}^{n} p\left(x_{i}, x_{i-1}\right)
$$

and its mesh is $\max p\left(x_{i}, x_{i-1}\right)$. The collection of all chains beginning at $x$ and ending at $y$ is denoted $\Sigma_{x y}$, while the subcollection of $\Sigma_{x y}$ consisting of those chains of mesh $\leqslant \mu$ is denoted $\Sigma_{x y \mu \cdot}(X, p)$ is called $\mu$-chainable if $\Sigma_{x y \mu}$ is nonempty for every $x, y \in X$.

ThEOREM 1. Let $\mathrm{f}$ be a $\mu, \lambda$-u.l.c. on the $\mu$-chainable metric space $(\mathrm{X}, \mathrm{p})$. There exists a metric $\overline{\mathrm{p}}$ topologically equivalent to $\mathrm{p}$ under which $\mathrm{f}$ is a contraction. Also $(\mathrm{X}, \overline{\mathrm{p}})$ is complete whenever $(\mathrm{X}, \mathrm{p})$ is.

Theorem 1 can be regarded as an "explanation," from the viewpoint of remetrization, of a result in [2]; our proof for it is largely a rephrasing from this viewpoint of the corresponding argument in [2].

Corollary 1.1. If $\mathrm{f}$ is a local contraction on compact $(\mathrm{X}, \mathrm{p})$, then $\mathrm{f}$ is a u.l.c. on $(\mathrm{X}, \mathrm{p})$, and so theorem 1 applies if $(\mathrm{X}, \mathrm{p})$ is $\mu$-chainable for every $\mu>0 .^{3}$

THEOREM 2. Let $\mathrm{f}, a \mu$-u.l.c. on the $\mu$-chainable metric space $(\mathrm{X}, \mathrm{p})$, possess a fixed point $\xi$. Then for each $\mathrm{x} \in \mathrm{X}$, the sequence of iterates $\mathrm{f}^{\mathrm{n}}(\mathrm{x}) \rightarrow \xi$ with ultimately geometric convergence.

THEOREM 3. Under the conditions of Theorem 2, there is a metric $\overline{\mathrm{p}}$ topologically equivalent to $\mathrm{p}$ and complete if $\mathrm{p}$ is, such that $\mathrm{f}$ is a contraction on $(\mathrm{X}, \overline{\mathrm{p}})$.

THEOREM 4. Let $\mathrm{f}$, a local contraciion on a complete metric space $(\mathrm{X}, \mathrm{p})$, possess a fixed point $\xi$, and suppose for each $\mathrm{x} \in \mathrm{X}$, the sequence of iterates $\mathrm{f}^{\mathrm{n}}(\mathrm{x}) \rightarrow \xi$. Then there is a complete metric $\overline{\mathrm{p}}$ topologically equivalent to $\mathrm{p}$ such that $\mathrm{f}$ is a contraction on $(\mathrm{X}, \overline{\mathrm{p}})$.

\section{Proofs of Theorems; Examples}

\subsection{Proofs of Theorem 1 and Corollary 1.1}

To prove theorem 1, we introduce

$$
\bar{p}(x, y)=\inf \left\{L\left(\sigma_{x y}: \sigma_{x y} \in \Sigma_{x y \mu}\right\},\right.
$$

which is well-defined for all $x, y \in X$ because $(X, p)$ is $\mu$-chainable, and obeys (4) because $\sigma_{x y}$ 's are just $\sigma_{y x}$ 's read backwards. The triangle inequality (3) holds for $\bar{p}$ because given $x, y, z \in X$ and any $\delta>0$, we can choose a $\sigma_{x y}$ and a $\sigma_{y z}$ for which

$$
L\left(\sigma_{x y}\right)<\bar{p}(x, y)+\delta, L\left(\sigma_{y z}\right)<\bar{p}(y, z)+\delta ;
$$

\footnotetext{
${ }^{3}$ A first proof of this was given by J. Levy of the Operations Research Section, NBS.
}

following $\sigma_{x y}$ with $\sigma_{y z}$ gives a $\sigma_{x z}$ for which we have

$$
\bar{p}(x, z) \leqslant L\left(\sigma_{x z}\right)=L\left(\sigma_{x y}\right)+L\left(\sigma_{y z}\right)
$$

$$
\leqslant \bar{p}(x, y)+\bar{p}(y, z)+2 \delta .
$$

The triangle inequality for $p$ shows that $\bar{p}(x, y)$ $\geqslant p(x, y)$, and so $\bar{p}$ satisfies the "positive definiteness" requirement (1). To complete the proof that $(X, \bar{p})$ is a metric space, we show that $\bar{p}(x, x)=0$ by setting $x=y$ in

$$
\bar{p}(x, y)=p(x, y) \quad \text { if } p(x, y) \leqslant \mu,
$$

which is true because the chain $[x, y] \epsilon \Sigma_{x y \mu}$ if $p(x, y)$ $\leqslant \mu$. Since $\bar{p}(x, y) \geqslant p(x, y),(7)$ also applies whenever $\bar{p}(x, y) \leqslant \mu$.

From (7) we see that $p$ and $\bar{p}$ are "identical in the small" and thus surely topologically equivalent; also that $(X, \bar{p})$ and $(X, p)$ have the same Cauchy sequences, so that the first is complete if the second is.

It only remains to show that $f: X \rightarrow X$, which by hypothesis is a $\mu, \lambda$-u.l.c. on $(X, p)$, is a contraction on $(X, \bar{p})$. To any chain

$$
\sigma_{x y}=\left[x=x_{0}, x_{1}, \ldots, x_{n}=y\right] \epsilon \Sigma_{x y}
$$

we can associate the chain

$$
f\left(\sigma_{x y}\right)=\left[f(x)=f\left(x_{0}\right), f\left(x_{1}\right), \ldots, f\left(x_{n}\right)=f(y)\right] \epsilon \Sigma_{f(x) f(y)} .
$$

Because $\lambda<1$ and $f$ is a $\mu, \lambda$-u.l.c., we have

$$
\sigma_{x y} \in \Sigma_{x y \mu} \text { implies } f\left(\sigma_{x y}\right) \epsilon \Sigma_{f(x) f(y) \mu} \text {. }
$$

For any $x, y \in X$ and any $\delta>0, \sigma_{x y} \in \Sigma_{x y \mu}$ can be chosen so that

$$
L\left(\sigma_{x y}\right) \leqslant \bar{p}(x, y)+\delta .
$$

Using the hypothesis on $f$ together with (8), we have

$$
\bar{p}(f(x), f(y)) \leqslant L\left(f\left(\sigma_{x y}\right)\right) \leqslant \lambda L\left(\sigma_{x y}\right) \leqslant \lambda \bar{p}(x, y)+\lambda \delta .
$$

Since $\delta$ is arbitrary, $f$ is indeed a contraction on $(X, \bar{p})$.

To prove the corollary, let $f$ be a local contraction on compact $(X, p)$, with associated functions $\mu(x)$ and $\lambda(x)$. The collection of open spheres

$$
\left\{S^{0}(x, \mu(x)): x \in X\right\}
$$

covers $X$, and so some finite subcollection

$$
\left\{S^{0}\left(x_{i}, \mu\left(x_{i}\right)\right): 1 \leqslant i \leqslant n\right\}
$$

also covers $X$. Let

$$
\lambda=\max _{i} \lambda\left(x_{i}\right)
$$

For each $x \epsilon X$, set

$$
\mu^{\prime}(x)=\min _{i}\left\{\mu\left(x_{i}\right)-p\left(x_{i}, x\right): x \epsilon S^{0}\left(x_{i}, \mu\left(x_{i}\right)\right)\right\} .
$$


Then if $y, z \epsilon S^{0}\left(x, \mu^{\prime}(x)\right)$ it follows that $y, z \epsilon S^{0}\left(x_{i}, \mu\left(x_{i}\right)\right)$ for some $i$ and therefore that

$$
p(f(y), f(z)) \leqslant \lambda\left(x_{i}\right) p(y, z) \leqslant \lambda p(y, z) .
$$

Thus $f$ is a $\lambda$-u.l.c. on $(X, p)$ using $\mu^{\prime}(x)$; since $\mu^{\prime}(x)$ is a continuous positive-valued function on the compact space $(X, p)$, it has a positive minimum $\mu_{0}$. Then $f$ is a $\mu_{0}, \lambda$-u.l.c. on $(X, p)$, as desired.

Note that when theorem 1 is applicable, the "geometric convergence" conclusion of the contraction theorem is maintained; that is, the contraction theorem applied to $(X, \bar{p})$ gives geometric convergence in $f^{n}(x) \rightarrow x_{0}$, but by virtue of (7) this implies "ultimately geometric convergence" in $(X, p)$.

The requirement of $\mu$-chainability is unnecessarily restrictive but we defer further elaboration until after the proofs of the remaining theorems.

\subsection{Examples}

Our first example is a simple illustration of theorem 1. Consider the circular arc described in the complex $z$-plane by

$$
X=\{\exp (i t): 0 \leqslant t \leqslant 3 \pi / 2\},
$$

and let $(X, p)$ consist of $X$ with the metric induced by that of the Euclidean plane. The map $f: X \rightarrow X$ given by

$$
f(\exp (i t))=\exp (i t / 2)
$$

is not a contraction since

$$
|\exp (i 3 \pi / 2)-\exp (0)|<|\exp (i 3 \pi / 4)-\exp (0 / 2)|,
$$

but it is easily shown to be u.l.c. Thus $(X, p)$ admits a remetrization which makes $f$ a contraction; the most obvious such metric, which is in a sense the limit as $\mu \rightarrow 0$ of the one obtained from theorem 1 , is

$$
\bar{p}\left(\exp \left(i t_{1}\right), \exp \left(i t_{2}\right)\right)=\left|t_{1}-t_{2}\right| .
$$

The second example shows that if $(X, p)$ is connected but not compact, and if $f$ is only a $\mu$-u.l.c., then one cannot guarantee a remetrization under which the contraction theorem becomes applicable. Let $X=[1, \infty)$ with the usual metric $p(x, y)=|x-y|$, and let $f: X \rightarrow X$ be defined by

$$
f(x)=x+x^{-1} .
$$

Then $f$ has no fixed point, and so cannot be made into a contraction on a complete $(X, \bar{p})$. To show that $f$ is a $\mu$-u.l.c. for arbitrary $\mu>0$, we observe that if $y$, $z \epsilon S(x, \mu)$ with $y<z$, then

$$
\begin{aligned}
|f(z)-f(y)| & =\left|z+z^{-1}-y-y^{-1}\right| \\
= & |z-y| \cdot\left|1-(y z)^{-1}\right| \leqslant|z-y| \cdot\left|1-(x+\mu)^{-2}\right|,
\end{aligned}
$$

so that $\lambda(x)=1-(x+\mu)^{-2}$ works.
Analogously, a $\lambda$-u.l.c. on a complete connected metric space may not admit application of the contraction theorem after any remetrization. Examples, too lengthy to repeat here, are given in [3], [4].

\subsection{Proof of Theorem 2}

Let $\xi$ be a fixed point of $f$, a $\mu$-u.l.c. of $(X, p)$ with associated function $\lambda(x)$. We shall show that for any $x \in X$, there is a positive integer $k(x)$ such that $f^{k(x)}(x) \epsilon S(\xi, \mu)$. For $k \geqslant k(x)$, assuming $f^{k}(x) \epsilon S(\xi, \mu)$, we will then have

$$
p\left(f^{k+1}(x), \xi\right) \leqslant \lambda(\xi) p\left(f^{k}(x), \xi\right)
$$

and so $f^{k+1}(x) \epsilon S(\xi, \mu)$. Thus for $k \geqslant k(x)$

$$
p\left(f^{k}(x), \xi\right) \leqslant[\lambda(\xi)]^{k-k(x)} p\left(f^{k(x)}(x), \xi\right)
$$

and the theorem follows.

Given $x \in X$, choose $\sigma_{\xi x} \epsilon \Sigma_{\xi x \mu}$ with mesh $\tau<\mu$. If none exists then consider $f\left(\sigma_{\xi x}\right)$ which certainly has mesh less than $\mu$, and proceed as below. Let the points of this chain be $\xi=x_{0}, x_{1}, x_{2}, \ldots ., x_{n}=x$. Choose $j$ so large that if $\lambda=\lambda(\xi)$, then

$$
\lambda^{j} \mu<(\mu-\tau) / 2 \text {. }
$$

Then since $x_{1} \epsilon S(\xi, \mu)$, we have

$$
p\left(f^{j}\left(x_{1}\right), \xi\right)<(\mu-\tau) / 2
$$

and $f^{j}\left(x_{1}\right)$ precedes $f^{j}\left(x_{2}\right)$ in the chain $f^{j}\left(\sigma_{\xi x}\right)$ which runs from $\xi$ to $f^{j}(x)$. Also

$$
p\left(f^{j}\left(x_{1}\right), f^{j}\left(x_{2}\right)\right)<\tau ;
$$

from this and (9) it follows that $f^{j}\left(x_{2}\right) \epsilon S(\xi, \mu)$. Then

$$
p\left(f^{2 j}\left(x_{2}\right), \xi\right)<\lambda^{j} p\left(f^{j}\left(x_{2}\right), \xi\right)<(\mu-\tau) / 2
$$

and $f^{2 j}\left(x_{2}\right)$ precedes $f^{2 j}\left(x_{3}\right)$ in the chain $f^{2 j}\left(\sigma_{\xi x}\right)$. Arguing as above leads to $f^{2 j}\left(x_{3}\right) \epsilon S(\xi, \mu)$, and so on; we obtain $f^{(n-1) j}(x)=f^{(n-1) j}\left(x_{n}\right) \epsilon S(\xi, \mu)$ and so can take $k(x)=(n-1) j$.

\subsection{Proof of Theorem 3}

This proof is based on a construction due to L. Janos [5]. We begin by setting

$$
p_{1}(x, y)=\inf \left\{L\left(\sigma_{x y}\right): \sigma_{x y} \in \Sigma_{x y \mu}\right\} ;
$$

from the proof of theorem 1 it follows that $p_{1}$ is a metric topologically equivalent to $p$. Moreover $p_{1}(x, y)$ $=p(x, y)$ if $p(x, y)<\mu$ and in particular this holds if $x, y \in S(\xi, \mu / 2)$. It need not be the case that $f$ is a contraction on $\left(X, p_{1}\right)$. However, because $f$ is a $\mu$-u.l.c. we have $f\left(\Sigma_{x y \mu}\right) \subset \Sigma_{f(x) f(y) \mu}$ and so we can at least deduce that $f$ is nonexpanding on $\left(X, p_{1}\right)$, i.e.,

$$
p_{1}(f(x), f(y)) \leqslant p_{1}(x, y) .
$$


Again if $x, y \epsilon S(\xi, \mu / 2)$ then we even have

$$
\begin{aligned}
p_{1}(f(x), f(y))=p(f(x), f(y)) & \\
& \leqslant \lambda(\xi) p(x, y)=\lambda(\xi) p_{1}(x, y) .
\end{aligned}
$$

For the second step in the construction, define

$$
C_{0}=S(\xi, \mu / 2)
$$

and set $C_{1}=f^{-1}\left(C_{0}\right), C_{2}=f^{-1}\left(C_{1}\right)=f^{-2}\left(C_{0}\right)$, etc. Then $f\left(C_{j+1}\right) \subset C_{j}$ for $j=1,2, \ldots$, and by theorem 2

$$
X=\bigcup_{0}^{\infty} C_{j}
$$

For all $x$, define

$$
n(x)=\inf \left\{j: x \in C_{j}\right\} .
$$

By theorem 2 this is well defined. Next set

$$
c(x, y)=\max \{n(x), n(y)\},
$$

and

$$
\lambda=\lambda(\xi)
$$

Finally, set

$$
p_{2}(x, y)=\lambda^{-c(x, y)} p_{1}(x, y) .
$$

Then $p_{2}$ satisfies the requirements for a metric except possibly the triangle inequality. In particular

$$
p_{2}(x, y) \geqslant p_{1}(x, y) \text {. }
$$

Before proceeding to the third step we show that

$$
p_{2}(f(x), f(y)) \leqslant \lambda p_{2}(x, y) .
$$

In fact this follows from (11) and (12) for $x, y \in C_{0}$, and otherwise from the observation that $n(f(x))=n(x)-1$, if $x \in X-C_{0}$, so that $c(f(x), f(y))=c(x, y)-1$ if either $x$ or $y$ is in $X-C_{0}$.

For the third step, associate with each chain

$$
\sigma_{x y}=\left[x=x_{0}, x_{1}, \ldots, x_{n}=y\right]
$$

the length

$$
L_{2}\left(\sigma_{x y}\right)=\sum_{i=1}^{n} p_{2}\left(x_{i}, x_{i-1}\right)
$$

and define

$$
\bar{p}(x, y)=\inf \left\{L_{2}\left(\sigma_{x y}\right): \sigma_{x y} \in \Sigma_{x y}\right\} .
$$

We shall show that $\bar{p}$ is the metric required in theorem 3 .
First note that $\bar{p}(x, y) \geqslant p_{1}(x, y)$ follows from (13) and the triangle inequality for $p_{2}$. Clearly $\bar{p}(x, y)$ $=(\bar{p}(y, x), \bar{p}(y, x)>0$ if $x \neq y$, and $\bar{p}(x, x)=0$ because $p_{2}(x, x)=0$. That the triangle inequality (3) holds can be shown as in the proof of theorem 1 . Hence $(X, \bar{p})$ is a metric space. For each $\sigma_{x y} \in \Sigma_{x y}$ we have $f\left(\sigma_{x y}\right)$ $\epsilon \Sigma_{f(x) f(y)}$ and from (14) it follows that

$$
L_{2}\left(f\left(\sigma_{x y}\right)\right) \leqslant \lambda L_{2}\left(\sigma_{x y}\right) .
$$

Hence $\bar{p}(f(x), f(y)) \leqslant \lambda \bar{p}(x, y)$, i.e., $f$ is a contraction on $(X, \bar{p})$ with $\lambda=\lambda(\xi)<1$.

It remains to show the equivalence of $p$ and $\bar{p}$. Note first that $\bar{p}\left|C_{0} \equiv p\right| C_{0}$ which already guarantees, by theorem 2, that convergence to the fixed point $\xi$ is ultimately geometric. We already have $\bar{p}(x, y)$ $\geqslant p_{1}(x, y) \geqslant p(x, y)$ so that we merely need show that convergence to $x \in X-\{y: p(y, \xi)<\mu / 2\}$ with respect to $p$ implies convergence with respect to $\bar{p}$. We show this by demonstrating that for each point $x \in X-\{y$ : $p(y, \xi)<\mu / 2\}$ there is some sphere of positive radius centered at $x$, the points $y$ of which obey

$$
p_{2}(x, y) \leqslant \lambda^{-(n(x)+1)} p_{1}(x, y)=\lambda^{-(n(x)+1)} p(x, y) .
$$

To see this, notice that $f^{n(x)}(x) \epsilon C_{0}$ and hence $f^{n(x)+1}(x)$ $\epsilon\{y: p(y, \xi) \leqslant \lambda \mu / 2\}$. If not $x=f^{0}(x), x_{1}=f(x), x_{2}$ $=f\left(x_{1}\right)=f^{2}(x), \ldots, x_{n(x)+1}=f^{n(x)+1}(x)$, then by a simple continuity argument for $f^{n(x)+1}$ there exists a sphere $S=S(x, r(x))$ centered at $x$ with radius $r(x)$ and a sphere $S_{n(x)+1}=S\left(x_{n(x)+1}, \quad r\left(x_{n(x)+1}\right)\right)$ such that $S_{n(x)+1} \subset C_{0}$, and $f^{n(x)+1}(S) \subset S_{n(x)+1}$. Thus for $y \epsilon S, n(y) \leqslant n(x)+1$.

If now $p(x, y)<\min \{r(x), \mu\}$ then $p(x, y)=p_{1}(x, y)$ and (16) holds. Finally since $\bar{p}(x, y) \leqslant p_{2}(x, y)$ we have the desired result. It follows from the above that completeness is also preserved.

\subsection{Proof of Theorem 4}

As before let $C_{0}=\{y: p(y, \xi) \leqslant \mu(\xi) / 2\}, C_{j}=f^{-j}\left(C_{0}\right)$, $j>0$. Also define $n(x)$ and $c(x, y)$ as in theorem 3 . The first step in the construction of $\bar{p}$ is to define a metric $\hat{p}$ such that $f$ is nonexpanding on $(X, \hat{p})$ that is

$$
\hat{p}(f(x), f(y)) \leqslant \hat{p}(x, y) .
$$

To this end let

$$
\hat{p}(x, y)=\max \left\{p\left(f^{n}(x), f^{n}(y)\right), 0 \leqslant n\right\} .
$$

Note that this is well defined since $c(x, y)$ is finite. Also $\hat{p}$ satisfies (17). To show it is a metric notice that $\hat{p}(x, y) \geqslant p(x, y)$ and $\hat{p}(x, x)=0$. For the triangle inequality observe that for any $x, y$, and $z$ and for all $n \geqslant 0$,

$$
\begin{aligned}
p\left(f^{n}(x), f^{n}(y)\right) & \leqslant p\left(f^{n}(x), \mathrm{f}^{n}(z)\right)+p\left(f^{n}(z), f^{n}(y)\right) \\
& \leqslant \hat{p}(x, z)+\hat{p}(z, y)
\end{aligned}
$$

from which the result follows. 
It remains to demonstrate the equivalence of $\hat{p}$ and $p$. We have $\hat{p}(x, y) \geqslant p(x, y)$ and from the definition

$$
\hat{p}\left|C_{0}=p\right| C_{0} .
$$

Let $x \in X-\{y: p(y, \xi)<\mu(\xi) / 2\}$. (The continuity of the iterates $f^{j}$ guarantees that for any $\eta<\mu-p\left(f^{n(x)+1}(x), \xi\right)$ there is a $\delta>0$ such that $p(x, y)<\delta$ implies $p\left(f^{j}(x)\right.$, $\left.f^{j}(y)\right)<\eta$ for all $j \leqslant n(x)$ and hence for all subsequent $j$ as well). This then implies the equivalence of $\hat{p}$ and $p$ everywhere.

To complete the theorem define

$$
\tilde{p}(x, y)=\lambda(\xi)^{-c(x, y)} \hat{p}(x, y),
$$

and if $\tilde{L}\left(\sigma_{x y}\right)$ is defined analogously to $L\left(\sigma_{x y}\right)$ set

$$
\bar{p}(x, y)=\inf \left\{\tilde{L}\left(\sigma_{x y}\right): \sigma_{x y} \in \Sigma_{x y}\right\} .
$$

The proof is now a repetition of part of that of theorem 3.

\section{Extensions}

We can now elaborate on the remark made after theorem 1. We need not restrict ourselves to $\mu$ chainable spaces. It is sufficient to examine the $\mu$-chainable components, i.e., the maximal $\mu$-chainable subsets of any space and to treat several cases.

Formally let $\left\{C_{a}: a \in A\right\}$ be the collection of $\mu$-chainable components of $X$. A $\mu$-u.l.c. $f: X \rightarrow X$ induces a map $\bar{f}: A \rightarrow A$ as follows:

$$
\bar{f}(a)=\alpha \text { if } f\left(C_{a}\right) \subset C_{\alpha} .
$$

Notice that $\bar{f}$ is well defined since $f$ preserves $\mu$-chains. Let

$B_{1}=\{b \in A: \bar{f}(b)=b\}$,

$B_{k}=\left\{b \in A: \bar{f}^{k}(b)=b, \bar{f}^{j}(b) \neq b ; 1 \leqslant j<k\right\}(k=2,3, \ldots)$,

$A_{b}^{k}=\left\{a \in A: \overline{f^{j}}(a)=b \epsilon B_{k}\right.$ for some $j \geqslant 0$,

and $f^{\nu}(a) \notin B_{k}$ for $\left.\nu<j\right\}$,

$X_{b}^{k}=\cup\left\{C_{a}: a \epsilon A_{b}^{k}\right\}$

$C=\left\{a \epsilon A: \overline{f^{j}}(a) \epsilon A-\bigcup_{1}^{\infty} B_{k} ;\right.$ for all $\left.j \geqslant 0\right\}$.

Note that

$$
A=C \cup \bigcup_{k=1}^{\infty}\left\{A_{b}^{k}: b \epsilon B_{k}\right\} .
$$

We shall consider 3 cases. For a subset of the form $X_{b}^{1}$, we shall demonstrate a metric $p^{*}$ such that $f$ is a contraction on $\left(X_{b}^{1}, p^{*}\right)$, where here $f$ is either a $\mu, \lambda$ u.l.c. or a $\mu$-u.l.c. For a subset of the form $X_{b}^{k}$ with $k>1$, we shall show for a $\mu, \lambda$-u.l.c., that $f^{k}$ is a con- traction on a suitable subset and that $f$ has certain periodic properties. Finally we show that for all other subsets of $X$ no such results are possible.

We begin with subsets of the form $X_{b}^{1}$. Notice that by construction $X_{b}^{1}$ and $X_{b^{\prime}}^{1}$ are disjoint unless $b=b^{\prime}$. For definiteness we shall restrict attention to an $f$ which is a $\mu, \lambda$-u.l.c. on $(X, p)$ with the understanding that similar results hold for a $\mu$-u.l.c. We have then a collection of $\mu$-chainable components $\left\{C_{a} ; a \epsilon A_{b}^{1}\right\}$ such that $f\left(C_{b}\right) \subset C_{b}$ and $f^{j}\left(C^{a}\right) \subset C_{b}$ for each $a \in A_{b}^{1}$ and some $j=j(a) \geqslant 0$. By theorem $1, f \mid C_{b}$ is a contraction for the metric $\bar{p}: C_{b} \times C_{b} \rightarrow R$ (reals) defined by

$$
\bar{p}(x, y)=\inf \left\{L\left(\sigma_{x y}\right), \sigma_{x y} \in \Sigma_{x y \mu}\right\} .
$$

Thus there is some point $\xi \epsilon C_{b}$ such that $f(\xi)=\xi$. Let $S=\left\{x \in C_{b}: p(x, \xi) \leqslant \mu / 2\right\}$ and define as before $n(x)$ $=\inf \left\{j \geqslant 0: f^{j}(x) \epsilon S\right\}$. Notice that $f^{j}\left(C_{a}\right) \subset C_{b}$ for each $C_{a}$ and for some $j$ so that $n(x)$ is well defined. Also let $c(x, y)=\min \{n(x), n(y)\}$.

As a second step extend the definition of $\bar{p}$ as widely as possible. That is, define $\bar{p}(x, y)$ as above wherever $\Sigma_{x y \mu}$ is not empty. This of course is true for $x, y$ if $x$ and $y$ are in the same $\mu$-component $C_{a}$. Let now

$$
p^{\prime}(x, y)=\min \{\mu, \vec{p}(x, y)\}
$$

with the understanding that $p^{\prime}(x, y)=\mu$ if $\bar{p}(x, y)$ is undefined, and

$$
p^{\prime \prime}(x, y)=\lambda^{c(x, y)} p^{\prime}(x, y) .
$$

We remark that $p^{\prime \prime}$ again has the metric properties except possibly (3). Moreover exactly as in theorem 3

$$
p^{\prime \prime}(f(x), f(y)) \leqslant \lambda p^{\prime \prime}(x, y) .
$$

Finally if we define $L^{\prime \prime}\left(\sigma_{x y}\right)$ in analogy to $L\left(\sigma_{x y}\right)$ used earlier and set

$$
p^{*}(x, y)=\inf \left\{L^{\prime \prime}\left(\sigma_{x y}\right): \sigma_{x y} \epsilon \Sigma_{x y}\right\}
$$

then $p^{*}$ has the desired properties. A proof of this would merely repeat arguments used in theorem 3 . This construction is also applicable to a $\mu$-u.l.c. because the only things required are that there exist a fixed point and that the iterates of all points of $C_{b}$ converge to the fixed point. This last follows since $f$, being a $\mu$-u.l.c. preserves $\mu$-chains and a proof like that of theorem 2 can be carried through.

To treat subsets of the form $X_{b}^{k}$ with $k>1$, notice first that $B_{k}$ and $B_{k^{\prime}}$ are disjoint unless $k=k^{\prime}$. If we consider a single $B_{k}$ we can further partition as follows: for $b \epsilon B_{k}$ let $b=b(0), \bar{f}(b)=\bar{f}(b(0))=b(1), \ldots$, $\bar{f}^{k-1}(b(0))=b(k-1), \quad \bar{f}^{k}(b(0))=b(0)$. This partitions $B_{k}$ into a class of disjoint subsets of indices each of cardinality $k$. We now further restrict our attention to one such subset $\beta_{k}$. In this case it is already true that $f$ cannot satisfy the conditions of theorem 2; namely, there is no fixed point. We thus restrict ourselves to $f$ being a $\mu, \lambda$-u.l.c. on $(X, p)$. It follows 
that $f^{k}$ is a $\mu, \lambda^{k}$-u.l.c. on $\left(C_{b(i)}, p\right)$; and by theorem 1 there is a metric $\bar{p}_{i}$ on $C_{b(i)}$ such that $f^{k}$ is a contrac tion on $\left(C_{b(i)}, \bar{p}_{i}\right)$ and hence $f^{k} \mid C_{b(i)}$ has a unique fixed point $\xi_{i}, i=0,1,2, \ldots, k-1$. On the other hand we also have that $f^{k}\left(f^{j}\left(\xi_{i}\right)\right)=f^{j}\left(f^{k}\left(\xi_{i}\right)\right)=f^{j}\left(\xi_{i}\right)$ and so $f^{j}\left(\xi_{i}\right)$ is a fixed point of $f^{k} \mid C_{b(l)}, l \equiv i+j(\bmod k)$. It follows that the fixed points of $f^{k}$ are a unique set of periodic points of $f$. Further for any other points $y$ in the components $C_{b(i)},\left\{f^{k n}(y)\right\} \rightarrow \xi_{i}$ as $n \rightarrow \infty$ and so $f^{k n+j}(y) \rightarrow \xi_{l}, \quad l=i+j(\bmod k)$. Finally for the components $\left\{C_{a}: a \in A_{h}^{k}, b \in \beta_{k}\right\}, f^{m}\left(C_{a}\right) \subset C_{b(i)}$ for some $m$ and some $i$, and hence for $x \in C_{a}, f^{m+k^{n+j}}(x) \rightarrow \xi_{l}$, $l \equiv i+j(\bmod k)$.

The third case involves those components $C_{a}$ such that $\overline{f^{j}}\left(C_{a}\right) \epsilon A-\bigcup_{1}^{\infty} B_{k}, j=0,1,2, \ldots$. It is immediate that neither $f$ nor any power of $f$ has any fixed points so that there is no possibility for a contraction map. Also, since the $C_{a}$ are $\mu$-components, limit points of $\cup\left\{C_{a}: a \epsilon C\right\}$ are limit points of individual components and so the components do not converge to any limit. The following is an example of this last case:
Let $X$ be the subset of euclidean 2 -space which consists of the closed disks $C_{n}$ centered at $\left(2^{n}, 0\right)$ and with radii $2^{-n}, n=1,2, \ldots$. Let $f: C_{n} \rightarrow C_{n+1}$ be defined by

$$
f(x, y)=\left(\frac{x-2^{n}}{2}+2^{n+1}, \frac{y}{2}\right) .
$$

Then $f$ is a $\left(\frac{1}{2}, 1\right)$ - u.l.c.

\section{References}

[1] Kolmogorov and Fomin, Elements of the Theory of Functions and Functional Analysis 1, 43-45 (Rochester, Graylock, 1957).

[2] Edelstein, M., An extension of Banach's Contraction principle, AMS Proc. 12, No. 1, 7-10 (1961).

[3]. Rakotch, E., A note on 2-locally contractive mappings, Bull Res. Counc. of Israel 10, F4, 188-191 (1962).

[4] Naimpally, S., A note on contraction mappings, Nederl. Akad. Wetensch. Proc. Ser. A67, 275-279 (1964).

[5] Janos, L., Two operations on distance functions. Notices AMS 11. No. 5, 614-7 (Aug. 1964).

(Paper 69B3-151) 